\title{
Spaced-Based Multi-Sensor Maneuvering Group Targets Refined Tracking Algorithm
}

\author{
Haipeng Wang*, Xueyuan Lin, Tiantian Tang and Shuyi Jia \\ Institute of Information Fusion, Naval Aeronautical and Astronautical University, Yantai Shangdong, China \\ ${ }^{*}$ Corresponding author
}

\begin{abstract}
Aiming to solve the refined tracking problem of the maneuvering group targets with the space-based multi-sensor detections, an algorithm named multi-sensor refined tracking algorithm within maneuvering group targets based on patulous generalized S-D assignment (MSRT-MGT-PGSDA) is proposed. In this algorithm, based on the deep analysis of the characteristics of the maneuvering group targets with the spacebased multi-sensor detections, multiple feasibility division is obtained with the tracks and the measurements within the group targets. Moreover, the S-D assignment model is built with the basis of the cost function of each feasibility division. Finally the maneuvering model of every group targets is decided with the optimum feasibility division, and the state update of each targets in the group with the corresponding tracking model of the maneuvering group targets in reference [1]. The analysis results of the simulation data show that obvious advantages of this algorithm are established in the aspects of tracking accuracy, effective tracking rate and the real time, compared with the centralized interacting multiple model multi-sensor multiple hypothesis tracking algorithm which is a superior performance algorithm in the multi-sensor maneuvering target tracking field.
\end{abstract}

Keywords-manuvering tracking; group targets; refined tracking; patulous generalized $S-D$ assignment component

\section{INTRODUCTION}

In recent years, with the improvement of the space-based sensor resolution, extensive attention of scholars has been attracted towards the group targets tracking technique [1 3]. In some practical applications, compared to the whole trend of group targets, it tends to be more concerned about the individual targets within the group [2,3]

In the moving process of group targets, based on specific tactics or objective, the whole maneuver, including turn, climb, dive and so on will be occurred in group targets at any time. Moreover the specific group targets maneuvering patterns[1], namely, the splitting, merging and dispersing are occurred. In this case, the relative positions structure of group targets measurement occurs to zoom, shear or rotation transformation. Accordingly resolution state of the individual target in a group is more complex, the measurement complexity of maneuvering group targets is considered insufficiently in the traditional multi-sensor maneuvering group targets tracking technique[4 7], including adjustable white noise, interacting multiple model, Jerk model and so on. In addition, the existing maneuvering group target tracking algorithms[8 11] usually are concentrated on the position, direction, track history or other aspects to clear logical relationship with group splitting, merging or cross. Then the processing of maneuvering group targets is completed based on PDA[8] model, pattern space [9], MCMC particle filter[10], SMC-PHDF[11] or other methods. As a whole the algorithm still is concentrated on the whole group, the changes of maneuvering group targets tracking is few studies, moreover, the tracking of maneuvering group targets with the space-based multi-sensor detection is no study.

\section{THE MSRT-MGT-PGSDA ALGORITHM}

\section{A. Establishment of the Basic Model}

The $i_{s}$ measurement of the sensor $s$ is defined as

$$
\boldsymbol{z}_{\mathrm{si}_{s}}=\left\{\begin{array}{l}
\boldsymbol{m}_{\mathrm{st}}+\boldsymbol{\omega}_{s_{s}}, \text { if it is derived from the objects } \\
\boldsymbol{a}_{s_{s}}, \text { if it is derived from the clutter }
\end{array}\right.
$$

where $\boldsymbol{m}_{\text {st }}$ is the measurement obtained by the sensors; $\boldsymbol{m}_{s t}=H_{S}\left(\omega_{t}, \omega_{s}\right)$, namely, $\boldsymbol{m}_{s t}$ is obtained by nonlinear transform of true position vector $\omega_{t}$ or the sensor position $\omega_{s} ; \omega_{s i_{s}}$ is the measurement noise, $\omega_{s i_{s}} \sim \boldsymbol{N}\left(0, \boldsymbol{R}_{s}\right)$. It is assumed that the measurement noise of each sensor is independent, so the density of the false measurement $a_{s i_{s}}$ is defined as

$$
f_{a_{s_{s}}}(a)=\frac{1}{\psi_{s}}
$$

where $\psi_{s}$ is the observation area of the sensor $s$.

Set the integrated measurement set obtained by fusion center until the $K$ time is defined as

$$
\begin{gathered}
\boldsymbol{Z}^{K}=\{\boldsymbol{Z}(k)\}_{k=1}^{K} \\
\boldsymbol{Z}(k)=\left\{\boldsymbol{z}_{s i_{s}}(k)\right\}_{i_{s}=1}^{m_{s}(k)} \quad s=1, \ldots N_{s}
\end{gathered}
$$

where $N_{s}$ is the number of sensors; $m_{s}(k)$ is the number of the measurement obtained by the sensor $\mathrm{s}$ at $\mathrm{k}$ time. 
Based on $\boldsymbol{Z}(k)$, segmentation of the groups is accomplished by means of cycle threshold method. $\boldsymbol{U}_{j}$ is set as the jth group obtained. According to different sources of sensors of each measurement within the groups, which is expressed as

$$
\boldsymbol{U}_{j}=\left\{\boldsymbol{z}_{s_{s}}\right\}_{i_{s=1}^{\hat{m}_{s}^{j}}}^{\hat{m}^{j}} \quad s=1, \ldots \widehat{S}_{j}
$$

where $\hat{S}_{j}$ is the number of sensors of the measurement within $\boldsymbol{U}_{j} ; \hat{m}_{s}^{j}$ is the number of the measurements sourced by sensors within $\boldsymbol{U}_{j}$.

If all measurements within $\boldsymbol{U}_{j}$ are false measurement, $\boldsymbol{U}_{j}$ is defined as false group. The false measurement is assumed to be mutual independence and independent to the real measurement values, so the probability of that $\boldsymbol{U}_{j}$ is false group is

$$
f\left(\boldsymbol{U}_{j}\right)=\boldsymbol{a}_{j}^{U}=\prod_{s=1}^{\hat{s}_{j}}\left(\frac{1}{\psi_{s}}\right)^{\bar{m}_{s}^{j}}
$$

In order to offset the inadequacies of group measurement and group target association caused by the leakage test, then for a false group $\boldsymbol{U}_{s 0}$ is added for the group test set obtained by each sensor. Hence, group measurement from the sensor $s$ and the whole detection area were respectively expressed as

$$
\boldsymbol{U}_{s}=\left\{\boldsymbol{U}_{s i_{s}}\right\}_{i_{s}=0}^{m_{s}^{U}}, \boldsymbol{U}=\left\{\boldsymbol{U}_{s}\right\}_{s=1}^{N_{s}}
$$

where $m_{s}^{U}$ is the number of group measurement detected by the sensor $s$.

\section{Partition OF THE MEASUREMENTS OF THE GROUP TARGETS}

The three sensors are considered at first. $\boldsymbol{U}_{i_{1} i_{2} i_{3}}=\left\{\boldsymbol{U}_{s i_{s}}\right\}_{s=1}^{3}$ is assumed as group measurement of the same group target corresponding to $\boldsymbol{U}_{t}$ in three sensors, thus the probability function is

$$
P\left(\boldsymbol{U}_{i_{1} i_{2} i_{3}}\right)=\prod_{s=1}^{3}\left[P\left(\boldsymbol{U}_{s i_{s}}\right)\right]^{u\left(i_{s}\right)}\left[1-P_{D}^{s}\right]^{\hat{m}_{s}\left[1-u\left(i_{s}\right)\right]}
$$

where $\hat{m}_{s}$ is the number of measurements in the group $\boldsymbol{U}_{s_{s}} ; u\left(i_{s}\right)$ is index function, if $i_{s}=0, u\left(i_{s}\right)=0$, otherwise $u\left(i_{s}\right)=1 ; P\left(\boldsymbol{U}_{s i_{s}}\right)$ is the probability of a true group target corresponding to the group $\boldsymbol{U}_{s i_{s}}$, and

$$
P\left(\boldsymbol{U}_{s i_{s}}\right)=\prod_{i=1}^{\hat{m}_{t}}\left[P_{D}^{s} f\left(z_{i}^{s i_{s}} \mid \omega_{t}\right)\right]\left(1-P_{D}^{s}\right)^{\left(\hat{m}_{s}-\hat{m}_{t}\right)}
$$

where $\hat{m}_{t}$ is the number of measurements from true targets within $\boldsymbol{U}_{s i_{s}}$.

The possibility division is assumed as $\gamma=\left\{\boldsymbol{U}_{t}, \boldsymbol{U}_{f}\right\}$. Where $\boldsymbol{U}_{t}$ is the subset of group measurement association corresponding to group track; $\boldsymbol{U}_{f}$ is false group measure-ment subset, and $\boldsymbol{U}_{f}=\left\{\boldsymbol{U}_{s i_{s}} ; i_{s}=0,1,2, \ldots . \hat{m}_{s} ; s=1,2,3\right\}$.

$\boldsymbol{U}_{t}=\left\{\boldsymbol{U}_{s i_{s}}\right\}_{s=1}^{3}$, where $i_{s}$ is the subset combined by empty set or arbitrary elements of the set $\left\{1,2 \ldots . \hat{m}_{s}\right\} . i_{s}$ is no longer a single value, but a set, whose maximum number $\hat{m}_{s}^{\prime}$ is $\left(\prod_{i=1}^{\hat{m}_{s}} C_{\hat{m}_{s}}^{i}+1\right)$. For each sensor, the selection of $i_{s}$ is mutual independence. Therefore,

$$
\boldsymbol{U}_{t}=\left\{\boldsymbol{U}_{i_{1} i_{2} i_{3}} ; i=0,1,2 \ldots ., \hat{m}_{s}^{\prime} ; s=1,2,3\right\}
$$

The event is recorded as $\xi(\gamma)=\{\gamma$ is real $\}$, and $\Gamma=\{\gamma\}$ is set as all possible division. When the splitting, merging or dispersing is occurred in the group, the detecting state of group target within the sensor should be consistent from the view of group measurement. Therefore the number of $\gamma$ within $\Gamma$ can be cut based on the above principle. $\hat{\Gamma}=\{\gamma\}$ is set as possible division sets after being streamlined, whose maximum number is

$$
\widehat{m}_{s}^{\prime \prime}=\prod_{s=1}^{3} \sum_{i=1}^{\hat{m}_{s}} C_{\hat{m}_{s}}^{i}+1
$$

To determine which maneuvering is occurred for each group target at $k$ time, it is necessary to determine the corresponding relationship of group measurement and group track, namely, the optimal division is determined as $\gamma=\left\{\boldsymbol{U}_{t}, \boldsymbol{U}_{f}\right\}$ and the formula (12) needs to be solved.

$$
\max _{\gamma \in \Gamma} \frac{L(\gamma)}{L\left(\gamma_{0}\right)}
$$

where $\gamma_{0}=\left\{\boldsymbol{U}_{t}=\Phi, \boldsymbol{U}_{f}=\boldsymbol{U}\right\}$ is assumed that all group measurements are false group. 


$$
\begin{aligned}
& L(\gamma)=f[\boldsymbol{U} \mid \xi(\gamma)]
\end{aligned}
$$

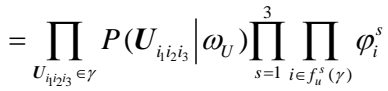

where $f_{U}^{s}(\gamma)$ is the label set of false group measurements of the sensor $\mathrm{s}$ within the division $\gamma ; \varphi_{i}^{S}$ is the probability of that the $i$-th group measurement in the sensor $s$ is false group measurement; $i_{1}, i_{2}$ and $i_{3}$ are respectively corresponded to the subset $\hat{i}_{1}, \hat{i}_{2}$ or $\hat{i}_{3} ; \omega_{U}$ is true position after the maneuvering occurred in the group; here the maximum likelihood estimation value $\hat{\omega}_{U}$ is replaced by $\omega_{U}$. Therefore

$$
\hat{L}(\gamma)=\prod_{U_{i 2 i_{3}=1}} P\left(\boldsymbol{U}_{i_{1} i_{2} i_{3}} \mid \hat{\omega}_{U}\right) \prod_{s=1}^{3} \prod_{i \in f_{u}^{s}(\gamma)} \varphi_{i}^{s}
$$

\section{Conformation of 3-D Assignment Problem}

The maximization problem described by the formula (12) is equivalent as follow.

$$
J^{*}=\min _{\gamma \in \Gamma} J(\gamma)=\min _{\gamma \in \Gamma}\left[\ln L\left(\gamma_{0}\right) \ln \hat{L}(\gamma)\right]
$$

The influence of $L\left(\gamma_{0}\right)$ is removed, thus

$$
J(\gamma)=\sum_{U_{h i 2 j} \in U} c_{i_{1} i_{2} i_{3}}
$$

$\rho_{i_{1} i_{3} i_{3}}$ is set as binary variable, and

$$
\rho_{i_{1} i_{2} i_{3}}= \begin{cases}1 & U_{i_{i} i_{2} i_{3}} \in \gamma \\ 0 & \text { otherwise }\end{cases}
$$

Because $\rho_{i_{1} i_{3} i_{3}}$ is one-to-one corresponding to the feasibility division $\gamma$, the generalized 3-D assignment problem of group target tracking can be described that

$$
J^{*}=\min _{\rho_{i 2} i 3} \sum_{i_{1}=0}^{\hat{m}_{1}} \sum_{i_{2}=0}^{\hat{m}_{2}} \sum_{i_{3}=0}^{\hat{m}_{3}} \rho_{i_{1} i_{2} i_{3}} c_{i_{1} i_{2} i_{3}}
$$

where $\hat{m}_{1}, \hat{m}_{2}$ and $\hat{m}_{3}$ are respectively the number of the feasibility division within three sensors. Constraint conditions are

$$
\begin{cases}\sum_{i_{2}=0}^{m_{2}} \sum_{i_{3}=0}^{m_{3}} \rho_{i_{1} i_{2} i_{3}}=1 & \forall i_{1}=1,2, \cdots \hat{m}_{1} \\ \sum_{i_{1}=0}^{m_{1}} \sum_{i_{3}=0}^{m_{3}} \rho_{i_{1} i_{2} i_{3}}=1 & \forall i_{2}=1,2, \cdots \hat{m}_{2} \\ \sum_{i_{1}=0}^{m_{1}} \sum_{i_{2}=0}^{m_{2}} \rho_{i_{i} i_{2} i_{3}}=1 & \forall i_{3}=1,2, \cdots \hat{m}_{3}\end{cases}
$$

\section{A. Conformation of Generalized S-D Assignment Problem}

When $N_{s}$ sensors are used to simultaneously observe the targets, S-D group vector needs to be structured for oriented group track in order to obtain the mapping relationship between group track and group measurement. Varies of feasibility division be found out, and the optimal partition is obtained by minimizing the cost of each feasibility division.

The generalized S-D assignment problem is constructed to

$$
J^{*}=\min _{\rho_{i_{2} 2-\cdots i_{s}}} \sum_{i_{1}=0}^{m_{1}=0} \sum_{i_{2}=0}^{m_{2}} \cdots \sum_{i_{N_{s}}=0}^{m_{N_{s}}} \rho_{i_{i} i_{2} \cdots i_{N_{s}}} c_{i_{1} i_{2} \cdots i_{N_{s}}}
$$

\section{Constraint conditions are}

$$
\begin{cases}\sum_{i_{2}=0}^{\hat{m}_{2}} \cdots \sum_{i_{N_{s}}=0}^{m_{N_{s}}} \rho_{i_{i}, \cdots i_{N_{s}}}=1 & \forall i_{1}=1,2, \cdots \hat{m}_{1} \\ \sum_{i_{1}=0}^{m_{1}} \cdots \sum_{i_{N_{s}}=0}^{\hat{m}_{N_{s}}} \rho_{i i_{1}, \cdots i_{N_{s}}}=1 & \forall i_{2}=1,2, \cdots \hat{m}_{2} \\ \cdots & \\ \sum_{i_{1}=0}^{m_{1}} \cdots \sum_{i_{N_{s}-1}=0}^{\tilde{m}_{N_{s}-1}} \rho_{i_{1} i_{2} \cdots i_{N_{s}}}=1 & \forall i_{N_{s}}=1,2, \cdots \widehat{m}_{N_{s}}\end{cases}
$$

where $\rho_{i_{1}, \cdots i_{N_{s}}}$ is binary association variable. If $\boldsymbol{U}_{i_{1} i_{2} \cdots i_{N_{s}}}$ in the feasibility division $\gamma$ is associated with a true group track, $\rho_{i_{i}, \cdots i_{N_{s}}}=1$, otherwise $\rho_{i_{1} i_{2} \cdots i_{N_{s}}}=0 . c_{i_{1} i_{2} \cdots i_{N_{s}}}$ is the cost function of the feasibility division, which can be deduced by means of 3-D assignment problem.

\section{B. State Update of the Tracks in the Formation}

The feasibility division $\gamma^{*}$ for $T$ group tracks is set to be at minimum cost, so $\gamma^{*}$ is most likely the division. Based on $\gamma^{*}$ can be obtained to group measurement set $\boldsymbol{Z}_{t}^{*}(k)$ of multiple sensor associated with group track $t$. The maneuvering pattern of group track $t$ is determined based on $\boldsymbol{Z}_{t}^{*}(k)$, and the corresponding group maneuvering model [1] is used to complete the state update of each track within the groups. 


\section{SimUlation VerificATION AND ANALYSIS}

\section{A. Simulation Environment}

The sensor is assumed to three $2 \mathrm{D}$ radar. In order to compare the performance of the two algorithms in different environments, the following two typical environments are provided. The environment 1 : the whole maneuver and splitting of the simulated group. There are 10 goals in the two dimensions, which is constituted to two groups. The first group is constructed of the former six targets. The environment 2: the merging and dispersing of the simulated group. The number of targets and constitution of the group is same to the environment 1 .

\section{B. Simulation Results}

The environment 1 :

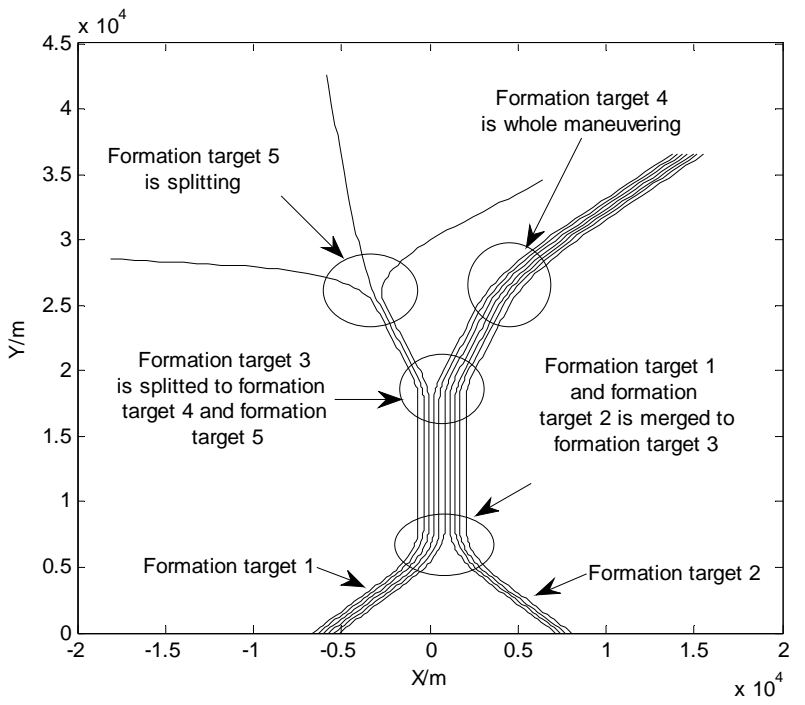

FIGURE I. TRUE STATE OF GROUP TARGETS (THE ENVIRONMENT 1)

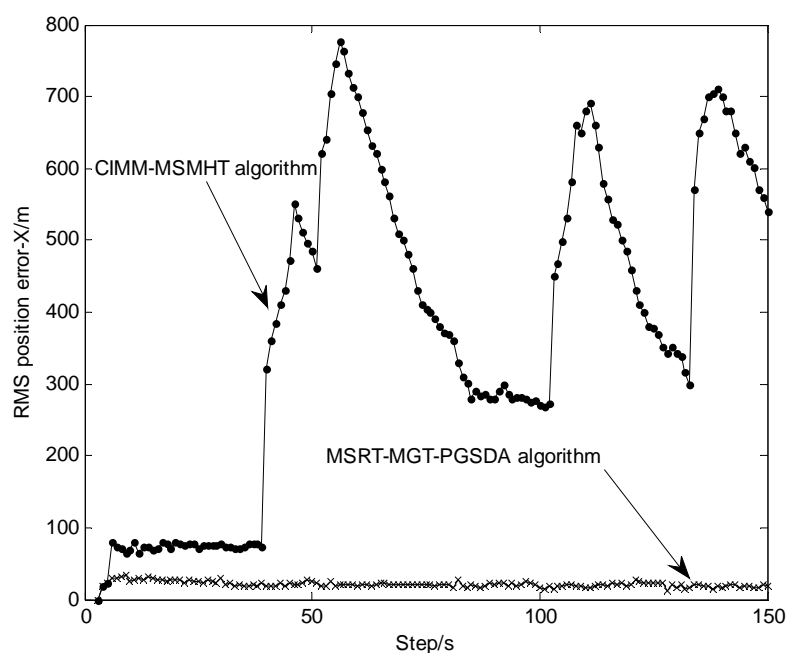

FIGURE II. RMS POSITION ERROR AT X-AXIS (THE ENVIRONMENT 1)

The environment 2 :

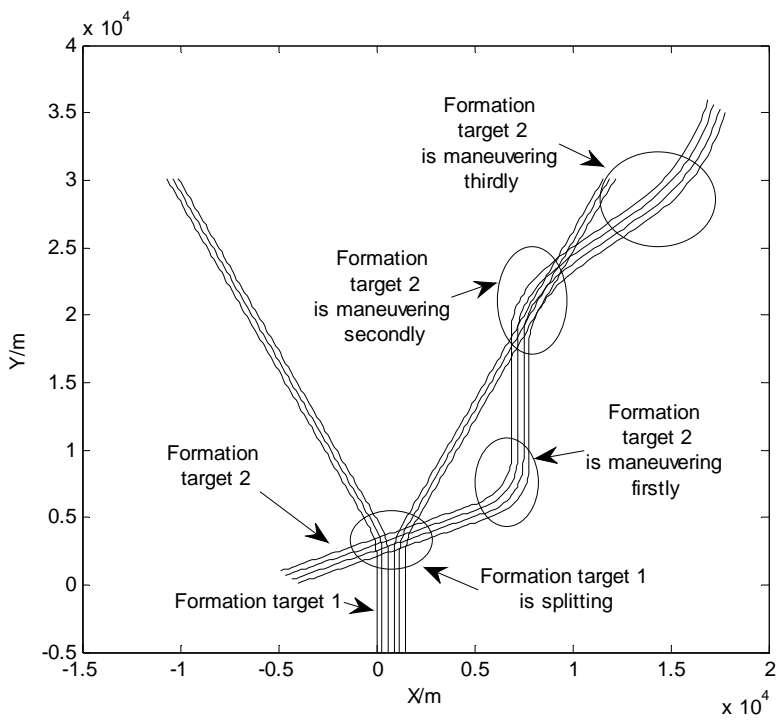

FIGURE III. TRUE STATE OF GROUP TARGETS(THE ENVIRONMENT 2)

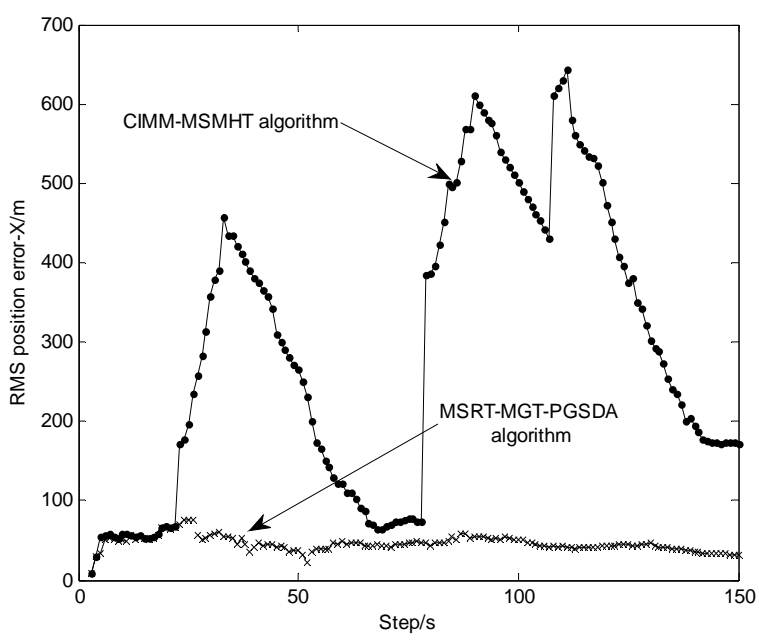

FIGURE IV. RMS POSITION ERROR AT X-AXIS(THE ENVIRONMENT 2)

\section{Simulation Analysis}

It can be seen from the Figure I that the splitting is occurred in group target 1 and the whole maneuver is occurred in group target 2 in the environment 1.And Figure III shows that the merging, splitting, dispersing and the whole maneuver are successively occurred in each group target in the environment 2. From the above all figures can be seen, compared with centralized interacting multiple model multisensor multiple hypothesis tracking (CIMM-MSMHT) algorithm[4] whose performance is superior in multi-sensor maneuvering target tracking algorithm, the RMS position error are most for CIMM-MSMHT algorithm, which is respectively as high as $650 \mathrm{~m}$ at part time. And his change is much bigger in the whole process, thus CIMM-MSMHT algorithm has been unable to meet the engineering requirements of tracking accuracy for the system. RMS position error for MSRT-MGTPGSDA algorithm is far less than CIMM-MSMHT algorithm, 
and the performance of MSRT-MGT-PGSDA algorithm is stable in the process of tracking.

The reason for the above results is: the maneuvering tracking model in CIMM-MSMHT algorithm is not matching, and the false association of point-to-track cross is easily occurred in clutter. Therefore RMS position error is all larger in several detection cycles of maneuvering group targets. Then a particular group maneuvering model is gradually adapted by automatically adjusting of the gate, false track elimination mechanism. RMS position error becomes small. Thus the change about RMS position error is much bigger. But the thought of multi-dimension assignment is used to affirm in real time the maneuvering situation of group targets in MSRTMGT-PGSDA algorithm, and the state updates are implemented by a specific tracking model of group targets. Therefore the values of RMS position error and his change in MSRT-MGT-PGSDA algorithm are far less than CIMMMSMHT algorithm from the whole tracking process.

\section{ACKNOWLEDGMENT}

This paper is supported by National natural science foundation of China (61531020), National natural science project (61471383), Equipment development fund (9140A07030514JB1400), and Equipment development fund(9140A07041415JB14001).

\section{REFERENCES}

[1] H. P. Wang. "Research on multi-sensor group targets tracking algorithm,” YT: Naval Aeronautical and Astronautical University,2012.

[2] Z. H. Peng, L. Sun, J. Chen. "Path Planning of Multiple UAVs Lowaltitude Penetration Based on Improved Multi-agent Co-evolutionary Algorithm," 2011 30th Chinese Control Conference, vol.7, pp. 40564061, 2011.

[3] Q. Zhen, C. R. Shelton. "Improving Multi-target Tracking via Social Grouping,” 2012 IEEE Conference on Computer Vision and Pattern Recognition, vol.7, pp. 1972-1978, 2012.

[4] Y. He, G. H. Wang, X. Guan. "Ingroup fusion theory with applications ,” BJ: Publishing House of Electronics Industry Press,pp.135-177,2011.

[5] M. Yu, H. Oh, W. H. Chen. "An improved multiple model particle filtering approach for maneuvering target tracking using airborne GMTI with geographic ingroup," Aerospace Science and Technology, vol.52,no.1,pp. 62-69,2016.

[6] W. Yang, Z. X. Wang, Y. W. Fu, et al. "Joint detection tracking and classification of a maneuvering target in the finite set statistics framework,” IET Signal Processing, vol.9,no.1,pp.10-20,2015.

[7] J. L. Yang, H. B. Ji, H. W. Ge. "Multi-model particle cardinalitybalanced multi-target multi-bernoulli algorithm for multiple maneuvering target tracking ," IET Radar, Sonar and Navigation, vol.7,no.2,pp.101-112,2013.

[8] W. D. Geng. "A study on group target merging and splitting method based on PDA," Journal of Radio Engineering, vol.37,no.2,pp. 2426,2007.

[9] H. Liu, W. D. Geng. “A Study of Merging and Splitting Methods for Group Targets Based on Pattern Space ,” Journal of Radio Engineering, vol.40,no.2,pp. 53-56,2010.

[10] F. Septier, S. K. Pang. “Tracking of Coordinated Groups Using Marginalized MCMC-Besed Particle Algorithm ," IEEE Transactions on Auto Control, vol.31,no.2,pp. 1-11,2009.

[11] F. Lian, C. Z. Han, W. F. Liu. "Sequential Monte Carlo Implementation and State Extraction of the Group Probability Hypothsis Density Filter for Partly Unresolvable Group Targets-tracking Problem ,” IET Radar, Sonar and Navigation, vol.4,no.5,pp. 685-702,2010. 